\title{
La pandemia COVID-19: lo que hemos aprendido hasta ahora desde España
}

\author{
Sergio Minué Lorenzo
}

\section{RESUMEN}

La pandemia COVID-19, producida por el virus SARS-CoV-2, ha producido un efecto devastador a nivel mundial, con efectos no antes observados en la vida de las personas y en la economía de los países. Esto paper analisa la respuesta del sistema sanitario español frente a la pandemia. Ademas, se proponie algumas recomendaciones para seguir adelante basadas en la revisión de la literatura publicada y en la propia evolución de la pandemia en España.

Palabras-clave: Pandemia COVID-19; Atención Primaria a Salud.

\section{ABSTRACT}

The COVID-19 pandemic, produced by the SARS-CoV-2 virus, has produced a devastating effect worldwide, with effects not previously observed in people's lives and in the countries' economies. This paper analyses the response of the Spanish health system to the pandemic. In addition, it gives some recommendations to approaching the COVID pandemic in Spain based on the review of the published literature and on the evolution of the pandemic in the country.

Keywords: Pandemic COVID-19; Primary Health Care.

Revista da Rede APS 2020

Publicada em: 15/04/2020

DOI:10.14295/aps.v2i1.66

Sergio Minué Lorenzo

(Escuela Andaluza de Salud Pública, España).

Correspondência para: Sergio Minué Lorenzo sergiominue21@gmail.com 


\section{LA MAGNITUD DEL PROBLEMA}

La pandemia COVID-19, producida por el virus SARS-CoV-2, ha producido un efecto devastador a nivel mundial, con efectos no antes observados en la vida de las personas y en la economía de los países. A 9 de abril de $2020^{1}$ había en el mundo más de un millón y medio de personas infectadas, de las cuales cerca de 90.000 habían muerto. Estados Unidos soportaba el mayor número de casos (435941) con casi 15000 muertes; España ocupaba el segundo lugar en número de casos (152446) y también en número de muertos (15238), desgraciado registro en el que Italia seguía ocupando el primer lugar con 17669 personas muertas. Brasil en ese momento tenía registrados 16275 casos con 826 muertos. Las tasas sin embargo eran significativamente diferentes, siendo los países con mayor número de personas infectadas por millón de habitantes España(3261) e Italia (2306) y en muertos por millón de habitantes también España (326) e Italia (392). Brasil aún presentaba tasas bajas, ya fuera en infectados por millón (77) o muertos por millón (4). Al existir también otros países con número de personas infectadas similares a España e Italia (como Alemania y Francia) pero con números absolutos y relativos bastante por debajo de los registros de Italia Y España, lo ocurrido en estos dos países puede ser de interés para otros países con el fin de reducir el impacto de la pandemia.

Si analizáramos la pandemia en función de su riesgo de desastre, éste sería directamente proporcional a la amenaza que representa y a la vulnerabilidad que existe frente a ella, e inversamente proporcional a la capacidad del sistema sanitario para hacerle frente. Severidad, vulnerabilidad y capacidad se analizan brevemente a continuación.

Aún no se dispone de información suficientemente contrastada para conocer la severidad de la infección por SARS-CoV-2. En principio, de los casos actualmente activos el $95 \%$ cursan de forma leve, mientras que sólo el
$5 \%$ presentan curso grave o severo. De los casos cerrados la tasa de letalidad (número de muertos respecto al número de infectados registrados) es actualmente del $21 \%$ cifra que ha ido aumentando cada mes. Al desconocerse la cifra real de personas infectadas es imposible conocer la tasa de fatalidad de la infección; el Imperial College ${ }^{2}$ estimaba que un $15 \%$ de la población española (rango 3,7-41\%) podría estar infectada. La CFR (Case fatality rate) de uno de los escasos experimentos naturales (el confinamiento del crucero Diamond Princess ${ }^{3}$ ) era del $1 \%$. Estos datos junto los resultados observados en lugares como Vo (Veneto) o Islandia, lleva a algunos investigadores a considerar que su letalidad no sería mucho mayor que la de la influenza.

Probablemente sea la vulnerabilidad especial frente al SARS-CoV-2 de determinadas poblaciones lo que eleve sustancialmente su severidad (con el consiguiente efecto de sobrecarga en el sistema sanitario), así como su mortalidad: estos factores son la edad y la existencia de enfermedades concomitantes (comorbilidades) especialmente enfermedades cardiocirculatorias, respiratorias o metabólicas. En España el 87,6\% de las personas muertas tenían más de 70 años ${ }^{4}$. Una posible explicación de la elevada tasa de fallecidos en España podría ser el elevado porcentaje de adultos mayores existentes en la población, al igual que en Italia. Sin embargo otros países con un porcentaje de población anciana tan elevado o mayor que en España, como Japón (el país más envejecido del mundo) registra solamente 94 muertos y una tasa por millón de 0.7. Lo que sí es un hecho significativo en España es que la mitad de las personas muertas residían en residencias de adulto mayor (7.300 de 14.500 a 8 de abril) ${ }^{5}$.

Finalmente la capacidad de buena parte de los sistemas sanitarios se ha visto claramente sobrepasada por las necesidades de atención de la pandemia; nuevamente Italia y España han mostrado sus carencias en este sentido: sí el número de camas de Unidades de Vigilancia Intensiva(UVI) de Italia representan un tercio de las existentes en Estados Unidos, el número de camas de cuidados intensivos antes de la 
pandemia era de 4500 públicas y 1000 privadas frente a 28.000 de Alemania que se convirtieron en 40.000 con la pandemia.

\section{LA RESPUESTA DEL SISTEMA SANITARIO}

\section{ESPAÑOL}

En el caso de España la respuesta sanitaria se ha centrado una vez más en los hospitales, que sigue siendo el verdadero centro de su sistema sanitario. La respuesta se realizó aumentando los recursos hospitalarios, tanto en camas como el personal sanitario. El refuerzo de los servicios de urgencia se acompañó de la redistribución de camas hospitalarias para atender a los pacientes afectos por COVID-19 y del incremento del número de camas en las Unidades de Cuidados Intensivos. Al producirse la saturación de los centros hospitalarios, y como segunda medida organizativa, se reconvirtieron en dispositivos hospitalarios otros tipo de instalaciones, como polideportivos y centros de convenciones, con capacidad potencial de albergar a 1000 o 2000 personas ( IFEMA en Madrid y la Fira en Barcelona).En el caso del IFEMA en Madrid (un palacio de congresos) existía una previsión inicial de dotarla de hasta 100 camas de cuidados intensivos que hubieron de ser reducidas al no disponerse de personas sanitario cualificado.

La puesta en marcha de estos centros precisaba de un número elevado de profesionales sanitarios, para lo cual se tomó la decisión de desplazar a médicos de familia y enfermería procedentes de los centros de Atención primaria que quedaron de esa forma desatendidos, bien porque vieron muy reducido su número de efectivos, o bien sencillamente porque fueron cerrados. En el caso de Cataluña supuso que más de la mitad de los centros de Atención Primaria fueron cerrados ${ }^{6}$, con lo que los ciudadanos dejaron de recibir atención domiciliaria, telefónica o en su centro sanitario de referencia. Similar situación se produjo en Madrid, la otra comunidad autónoma con mayor número de infectados.

La experiencia italiana puso de manifiesto claramente que uno de las causas del elevado número de casos y muertos sufridos, especialmente en el norte de Italia fue precisamente el centrar la respuesta en los hospitales, que actuaron además como fuente de infección para buena parte de la población.

Como señalaban Nacoti et al en New England ${ }^{7}$
"estamos aprendiendo que los hospitales pueden ser los principales vehículos de transmisión del covid-19. Rápidamente sobrecargados de pacientes infectados que pueden contagiar a los que no lo están. Los pacientes son transportados por nuestro servicio regional, que también contribuye a diseminar la enfermedad, convirtiendo rápidamente a profesionales y ambulancias en vectores. Los trabajadores sanitarios son vectores asintomáticos o enfermos sin vigilancia".

El trabajo aún más reciente de Boccia, Ricciardi y loannidis ${ }^{8}$ insiste en la necesidad de mantener los pacientes infectados por COVID-19 fuera de los hospitales salvo en caso de presentar síntomas severos. En España más del 15\% de los pacientes infectados son sanitarios, cifra mucho mayor que la existente en otros países aún con número elevado de casos, cifra coherente con las muy deficientes medidas de protección personal con las que han tenido que realizar su labor.

En un trabajo reciente se argumentaba $^{9}$ la necesidad de potenciar la Atención Primaria como recurso esencial de abordar la atención a los pacientes infectados: sus atributos esenciales (accesibilidad, continuidad de la atención, integralidad y coordinación de los servicios, junto a un enfoque familiar, comunitario y de orientación a las especificidades culturales) son aún más necesarios en situaciones de pandemia como demostró la epidemia de Ébola hace unos años. Nunca como en una situación de confinamiento social es más necesario mantener un contacto fácil y fluido de las personas con los profesionales que les atienden de forma cotidiana, ya sea para detectar tempranamente 
la infección, para realizar su seguimiento o para atender cualquier otro problema de salud, puesto que las personas siguen padeciendo otros problemas de salud que precisan de una respuesta integral; nunca es más necesario que en una pandemia realizar una adecuada coordinación de la atención prestada en los diferentes dispositivos del sistema, y nunca fue más importante el conocimiento acumulado de las personas y sus familias a lo largo del tiempo para conocer la situación en la que viven y tomar decisiones en condiciones de tanta incertidumbre. Como señalaba Nacoti et al "los sistemas sanitarios occidentales han sido construidos en torno al concepto de atención centrada en el paciente, pero una epidemia requiere un cambio de perspectiva hacia el concepto de atención centrada en la comunidad". El enfoque paradigmático de la Atención Primaria de Salud (APS).

\section{ALGUNAS RECOMENDACIONES PARA}

\section{SEGUIR ADELANTE}

En España la pandemia COVID-19 podría considerarse una infección de predominio nosocomial cuyo principal vector de transmisión son los propios profesionales sanitarios. Hospitales y especialmente residencias de adulto mayor han sido los principales focos de transmisión de la infección.

En este sentido algunas recomendaciones basadas en la revisión de la literatura publicada y en la propia evolución de la pandemia en España podrían ser la siguiente:

- Es imprescindible proteger al máximo los colectivos especialmente vulnerables, sobre todo adultos mayores y personas con enfermedades crónicas. Para ello se precisa aislarlos, conocer tanto si sus cuidadores como ellos mismos están infectados (para tomar las medidas de aislamiento $y$ seguimiento procedente en caso positivo), $y$ extremar la seguridad del contacto con las personas en contacto con ellos.

- Los profesionales sanitarios pueden representar la principal vía de transmisión del COVID-19; es esencial dotarlos de todos los medios de protección necesarios, conocer su estado frente al SARS-CoV-2, ponerles en aislamiento en caso positivo y no permitirles volver a atender pacientes hasta que su estado inmunológico lo permita.

- $\quad$ Si las medidas de aislamiento social son las más habitualmente recomendadas también deberían aplicarse a la hora de recibir atención sanitaria. Únicamente los pacientes más severos deberían recibir atención hospitalaria, asignando a la Atención Primaria la labor de detección precoz, identificación de casos, seguimiento, derivación, atención tras el alta hospitalaria y acompañamiento en la agonía si ésta se produce. Para ello APS debe poner en juego a todos sus profesionales y no exclusivamente a los médicos, con una doble orientación: distribución de funciones, y priorización de atención en domicilio y a distancia (teléfono, video, whatsapp). 


\section{NOTAS E REFERÊNCIAS}

${ }^{1}$ Worldometers. Consultado el 9 de abril de 2020 en: https://www.worldometers.info/coronavirus/

${ }^{2}$ Flaxman S, Mishra S, Gandy A, Unwin HJT, Coupland H, Mellan TA et al on behalf Imperial College COVID19 Response Team.Estimating the number of infections and the impact of non-pharmaceutical interventions on COVID-19 in 11 European countries. Imperial College. London.30 March 2020

${ }^{3}$ Ioannidis J. Coronavirus disease 2019: The harms of exaggerated information and non-evidence-based measures. European J Clinical Investigation 2020;50(4): April 2020. e13222

${ }^{4}$ Centro de Coordinación de Alertas y Emergencias Sanitarias Actualización no 70. Enfermedad por el coronavirus (COVID-19).09.04.2020. Ministerio de Sanidad. Gobierno de España.2020

${ }^{5}$ Nius. Consultado el 9 de abril en: https://www.niusdiario.es/sociedad/sanidad/coronavirus-recuentomuertos-residencias-ancianos-toda-espana-8500_18_2927670056.html

6 Ara. Consultado el 6 de abril en: https://www.ara.cat/societat/Mes-meitat-dels-CAP-tancatscoronavirus-covid-19_0_2430356950.html

${ }^{7}$ Nacoti M, Ciocca A, Giupponi A, Brambillasca P, Lussana F, Pisano M, et al. At the Epicenter of the Covid19 Pandemic and Humanitarian Crises in Italy: Changing Perspectives on Preparation and Mitigation. NEJM Catal. 2020. DOI: 10.1056/CAT.20.0080. Disponible en: https://catalyst.nejm.org/doi/pdf/10.1056/CAT.20.0080

${ }^{8}$ Boccia SS, Richiardi W, loannidis J. What Other Countries Can Learn From Italy During the COVID-19 Pandemic. JAMA Internal Medicine Published online April 7, 2020.

${ }^{9}$ Minué S. Contra el coronavirus, más Atención Primaria que nunca. AMF 2020;Consultado el 6 de abril en : https://amf-semfyc.com/web/article_ver.php?id=2626 